\title{
ANTINUCLEAR ANTIBODIES IN ENDOMETRIOSIS
}

\author{
Laís Zanlorenzi ${ }^{1,}$, Nelzi Ferreira de Queiroz Junior ${ }^{1}$, Carlos Gomes Bezerra Sobrinho ${ }^{1}$, Laura Vilas Boas ${ }^{1}$, Renato Nishiara ${ }^{1}$, \\ Thelma Skare ${ }^{1}$ \\ 1.Hospital Universitário Evangélico Mackenzie, Curitiba (PR), Brazil. \\ *Corresponding author: laisznl@gmail.com
}

\section{BACKGROUND}

Endometriosis is a chronic gynecological condition characterized by the presence of endometrial-like tissue outside the uterine cavity. Its prevalence in women of reproductive age is 2-10\% and reaches 35-50\% among women with pain and/or infertility. Although the etiology of endometriosis is complex and poorly elucidated, studies suggest strong associations between endometriosis and abnormalities in the immune system. The aim of this study was to determine the prevalence of antinuclear antibodies (ANA) and anti-DNA in patients diagnosed with endometriosis. To evaluate the association between the presence of ANA and/or anti-DNA and demographic factors of patients with endometriosis.

\section{METHODS}

An analytical cross-sectional study was conducted. The sample was composed of 94 patients with diagnosis of endometriosis confirmed by videolaparoscopy and biopsy with anatomopathological study. Demographic and clinical data was obtained using a questionnaire. As a control group we used 91 patients matched in age and sex. Antinuclear antibodies were searched by indirect immunofluorescence on HEp-2 cells, using the commercially available kit ANA HEp-2 (Zion, USA) and anti-dsDNA using Crithidia luciliae (Medivax, SP).

\section{RESULTS}

Of the 94 patients evaluated with endometriosis, $21.27 \%$ had positive ANA compared to 5.49\% of the control group ( $p<0.001$; $\mathrm{OR}=4.6 ; 95 \% \mathrm{IC}=1.6-13.0$ ). Fine speckled pattern was the most commonly seen (65\%) followed by fine dense speckled in $15 \%$, nucleolar in 10\%, homogeneous and coarse speckled with 5\% each. Titter ranged from 1:80 to 1:320. No associations of ANA with age, disease duration, number of pregnancies or abortions were found (all $p=n s$ ). Patients and controls were all negative for the anti-dsDNA test.

\section{CONCLUSION}

In the present study, patients with endometriosis have higher prevalence of ANA compared to the control. Patients with endometriosis did not show reactivity for anti-dsDNA antibody. Presence of ANA was not associated with demographic data or obstetrical history.

\section{KEYWORDS}

Antinuclear antibodies, Endometriosis, Anti-DNA antibodies, Autoimmunity, Inflammation. 